\title{
Urachal remnants presenting as an umbilical sinus in middle age: a rare case report
}

\author{
Shruthi Karishma, S. J. Haridarshan*, S. Rajagopalan
}

Department of General surgery, Rajarajeswari Medical College Hospital, Bangalore, Karnataka, India

Received: 11 November 2017

Accepted: 09 December 2017

*Correspondence:

Dr. S. J. Haridarshan,

E-mail: sjharidarshan@gmail.com

Copyright: (C) the author(s), publisher and licensee Medip Academy. This is an open-access article distributed under the terms of the Creative Commons Attribution Non-Commercial License, which permits unrestricted non-commercial use, distribution, and reproduction in any medium, provided the original work is properly cited.

\begin{abstract}
Urachal sinus is a rare type of Vitello intestinal duct anomalies. Clinical presentation is that of persistent umbilical discharge. It is usually seen in paediatric age group. Moreover, they have a different course in adults than paediatric age group in which they frequently involute and have a benign course. These remnants are prone to infection and development of malignancy. A proper diagnostic workup by clinical and imaging tools is required. Early removal of urachal remnants at first diagnosis is key for preventing future morbidities. In our case, all the three remnants (Vitello intestinal duct, urachus and ligamentum venosum) were found in a middle-aged patient and hence is unusual and a rare presentation.
\end{abstract}

Keywords: Omphalectomy, Sinogram, Urachus, Umbilical sinus, Vitello-intestinal duct

\section{INTRODUCTION}

Vitello intestinal duct or omphalomesenteric duct (OMD) is an embryonic structure, which connects the yolk sac to the midgut and failure of its resorption results in various anomalies including Meckel's diverticulum, patent vitelline duct, fibrous band, sinus tract, umbilical polyp and cyst. These anomalies occur in approximately $2 \%$ of the population and may remain silent throughout life, or may present incidentally with an intraabdominal complication. ${ }^{1}$ Umbilical disorders are frequently encountered in paediatric surgery. But, the presentation and progression in paediatric and adult population is different. Adults have a higher risk of urachal cancer and incur more morbidity. Thus, proper and early diagnosis of urachal pathologies is must. Due to rarity, urachal anomalies present a diagnostic challenge in adult population.

We report a middle-aged patient with a discharging umbilical sinus in whom all the three remnants of Vitello intestinal duct namely, obliterated Vitello intestinal duct, partially obliterated ligamentum venosum and patent urachus were present.

\section{METHODS}

A 55-year-old male presented with creamy foul-smelling discharge from umbilicus for the past 1 year which was at times blood tinged associated with pain over umbilicus. On examination, umbilicus was inverted and centrally placed with creamy non-blood-stained discharge and tenderness around the umbilicus. Contrast enhanced CT abdomen with a sinogram of umbilical sinus revealed multiple blind tracts opening into the umbilicus in the pre-peritoneal space, one extending upwards and the other towards the bladder ending blindly (Figure 1).

Culture and sensitivity of the discharge showed no signs of infection. A working diagnosis of umbilical sinus was made and after pre-operative assessment and preanaesthetic work up, patient was taken up for exploration 
of the sinus after an informed, written and signed consent.

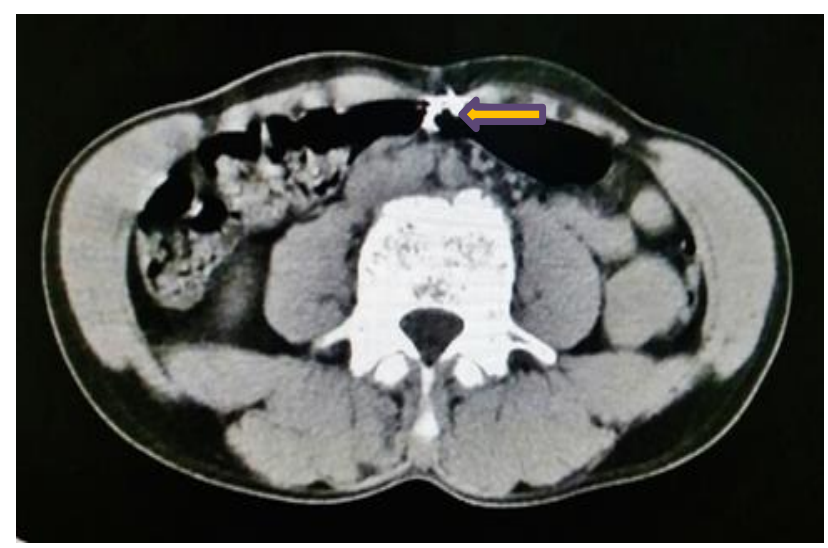

Figure 1: The umbilical sinus as pointed by the arrow in CT scan.

Intra-operative findings included umbilicus with obliterated urachus, Vitello-intestinal duct and obliterated umbilical vein. Patient underwent omphalectomy with excision of the sinus tract and all the three remnants of umbilicus (Figure 2,3).

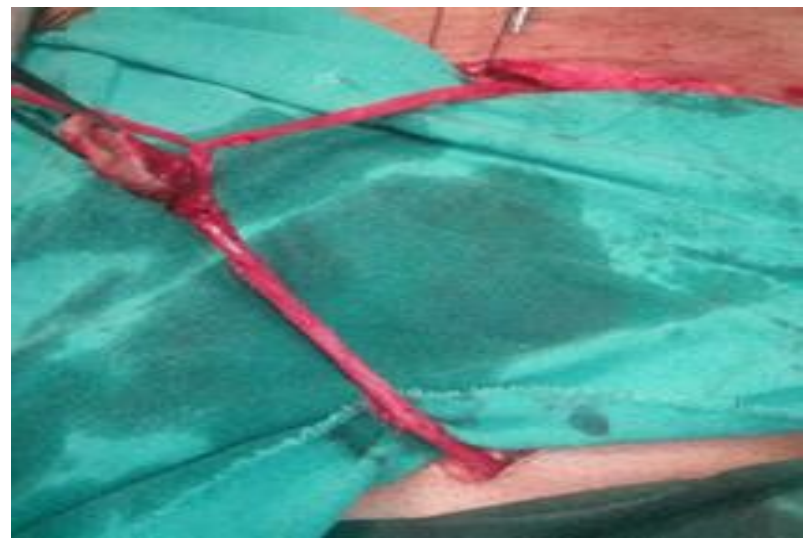

Figure 2: Demonstrating the urachal remnants arising from the umbilicus.

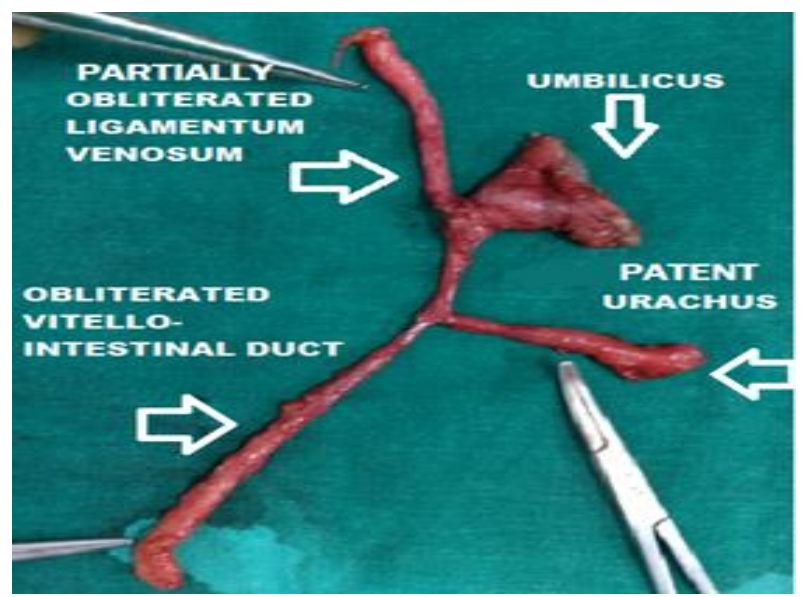

Figure 3: Complete specimen after resection.
Post-operative stay was uneventful. Histopathology of the specimen reported keratinized squamous epithelium of the remnants with no features of malignancy.

\section{DISCUSSION}

As far as umbilical discharge is concerned, it is quite alarming and in adults only a few studies on the urachal sinus have been conducted. Causes are congenital and acquired, though acquired conditions are more common.

The incidence of urachal pathologies in childhood is approximately 1 in 5000 with a male to female ratio of $3: 1^{2}$

In adults it is rare, approximately 2 cases per 100,000 hospital admissions, because urachal anomalies usually involute in early childhood. ${ }^{3}$

The presentation and progression in pediatric and adult population is different. Adults have a higher risk of urachal cancer and incur more morbidity. Thus, the proper and early diagnosis of urachal pathologies is must. Due to rarity, urachal anomalies present a diagnostic challenge in adult population. However, with proper Clinical and Imaging workup they can be managed effectively. ${ }^{4}$

Urine discharge from umbilicus suggests patent urachus, hematuria points to vesico urachal diverticulum and pus discharge from umbilicus may be present with urachal sinus. Further complications such as infection and malignancy may present. Imaging has a definitive role in classifying the type of urachal anomaly and further characterizing the disease. In case of external opening, contrast can be given through the umbilicus to delineate the tract.

A relatively anterior location in the preperitoneal space with no obscuration by bowel gas makes ultrasound a good tool for diagnosis. CT is further required for confirmation and to look for malignancy. Findings include a tract/collection extending from the umbilicus with prominent median umbilical vein. In case there is a tract communicating with the bladder then it signifies a patent urachus. Blind ending tract arising from umbilicus suggests umbilical sinus. And double-blind ending cavity is a urachal cyst. Urachal anomalies may get secondarily infected via lymphatic, hematogenous or vesical route by a wide spectrum of microorganisms and may form a urachal abscess. CT may reveal inhomogeneous attenuation or abnormal enhancement (rim or patchy enhancement)..$^{5}$

Treatment includes complete excision of the urachal remnant. In case of infection/abscess, initial control of infection/pus drainage should be followed by surgery. A complete excision of the wall is important as there is a high probability of reinfection and chances of development of malignancy in residual remnants. ${ }^{6,7}$ 


\section{CONCLUSION}

Urachal anomalies are rare clinical entities and asymptomatic urachal sinuses persisting into late adulthood even more so. Infected urachal remnant should be considered in patients with umbilical discharge and inflammation. Finding all the three remnants is an extremely uncommon anomaly. However, it should be considered as a differential diagnosis of discharging umbilicus. Correct diagnosis with multimodality imaging and complete surgical resection is recommended to prevent subsequent reinfection or malignant transformation.

Funding: No funding sources Conflict of interest: None declared

Ethical approval: Not required

\section{REFERENCES}

1. Sawada F, Yoshimura R, Ito K, Nakamura K, Nawata H, Mizumoto $\mathrm{K}$, et al. Adult case of an omphalomesenteric cyst resected by laparoscopicassisted surgery. World J Gastroenterol. 2006; $12: 825$.
2. Spataro RF, Davis RS, McLachlan MS, Linke CA. Barbaric Urachal abnormalities in the adult Radiology. 1983;149(3);659-63.

3. Yiee H, Garcia N, Baker LA, Barber R, Snodgrass WT, Wilcox DT. A diagnostic algorithm for urachal anomalies J Pediatr Urol. 2007;3(6):500-4.

4. Ashley RA, Inman BA, Routh JC, Rohlinger AL, Husmann DA, Kramer SA. Urachal anomalies: a longitudinal study of urachal remnants in children and adults. J Urol. 2007;178:1615-8.

5. Iuchtman M, Rahav S, Zer M, Mogilner J, Siplovich L. Management of urachal anomalies in children and adults Urol. 1993;42(4):426-30.

6. Goldman IL, Caldamone AA, Gauderer M. Infected urachal cysts: a review of 10 cases. J Urol. 1988;140:375-8.

7. Blichert-Toft M, Nielsen OV. Congenital patient urachus and acquired variants. Diagnosis and treatment. Review of the literature and report of five cases. Acta Chir Scand. 1971;137:807-14.

Cite this article as: Karishma S, Haridarshan SJ, Rajagopalan S. Urachal remnants presenting as an umbilical sinus in middle age: a rare case report. Int Surg J 2018;5:330-2. 